Article

\title{
Minimal decrease in triathlon running performance compared to isolated running performance is important for sprint triathlon success
}

\author{
Keigo Takahashi ${ }^{1,2}$ and Yoshiharu Nabekura ${ }^{3}$ \\ 1 Graduate school of Comprehensive Human Science, University of Tsukuba, Ibaraki 305-8574 Japan \\ 2 Japan Society for the Promotion of Science, Chiyoda-ku, Tokyo 102-8472, Japan \\ 3 Faculty of Health and Sport Sciences, University of Tsukuba, Ibaraki 305-8574, Japan
}

* Correspondence: tyro221@gmail.com

Received: 14 February 2021; Accepted: 08 June 2021; Published: 08 June 2021

\begin{abstract}
A strategy to improve running performance in a triathlon is minimizing fatigue from the preceding exercise. However, little is known about performance decrement, or the degree to which it changes, in triathlon running (TR) compared to isolated running (IR). This study examined the decrease in TR performance compared to IR performance and the degree of change as determined from sprint triathlon competition results. The data were obtained from the official result times of certification competition events from 2013 to 2016 (IR) and elite categories participating in the Asia Cup sprint triathlon from 2013 to 2016 (TR). Data of 236 athletes who finished IR and TR in the same year were analysed. The average performance was significantly worse in TR than in IR ( $<<0.01)$, and the effect size in a paired Wilcoxon test was large, regardless of competition years and sex. There were also large inter-individual differences in the performance decrement in TR compared with IR. The overall triathlon performance and performance decrement in TR compared with IR showed significant correlation (Spearman's rho $=0.47-0.76, \mathrm{p}<0.05$ ). In conclusion, TR performance was worse than the IR performance and the degree varied widely inter-individual. The smaller the performance decrement in TR compared with IR, the more likely the athlete is to succeed in the sprint triathlon.
\end{abstract}

Keywords: Competition; Triathletes; Endurance; Fatigue

\section{Introduction}

Triathlons consist of swimming, cycling, and running, all completed in this sequential order. Triathletes reportedly experience breathing disorders and incoordination during the transition from cycling to running (Hue et al., 1999; Chapman et al., 2010). The ability of the triathlete to adapt through this transition is important for triathlon success (Sleivert \& Rowlands, 1996). The focus of triathlon studies has mainly been on the physiological and biomechanical responses to running after cycling or swimming and cycling (Millet \& Vleck, 2000).
There are notable increases in oxygen uptake (VO2), ventilation (VE), heart rate (HR), breathing frequency, blood lactate (BLa) concentration, $\mathrm{VO} 2$ of the respiratory muscles, and core temperature in triathlon running (TR; running preceded by cycling or swimming and cycling) when compared with isolated running (IR; without prior swimming and cycling) (Hue et al., 2000; Taylor \& Smith, 2013; Walsh et al., 2017; Walsh et al., 2015). From a biomechanical perspective, notable changes also occur in muscle activity, leg stiffness, and running kinematics in TR when compared with IR (Walsh et al., 2017; Le Meur et al., 2012; Rendos et al., 2013). Therefore, it is commonly considered that the performance 
decrement in TR compared with IR is due to physiological and biomechanical changes following the preceding exercise.

Although studies on the general effects of cycling on subsequent running have been investigated the overall tendency of triathletes, recent studies have also begun focusing on inter-individual differences. Bonacci et al. (2010) compared IR and running after 45 minutes of high intensity cycling and reported that seven out of 15 triathletes demonstrated changes in muscle activity and running kinematics during running after cycling, when compared with IR. In addition, eight out of 15 triathletes demonstrated an increase or decrease in VO2. Similarly, du Plessis et al. (2020) also demonstrated the importance of assessing inter-individual responses to running after cycling. Other studies have identified possible factors that could also influence inter-individual responses, such as pacing strategy, drafting conditions, triathlon experience, and performance level (Wu et al., 2014; Hausswirth et al., 2010; Hausswirth et al., 2001; Rendos et al., 2013; Millet et al., 2000). Taken together, the effects of cycling on subsequent running vary interindividually. Accordingly, the performance decrement in TR compared with IR can also vary among individuals.

Many studies have provided physiological and biomechanical knowledge on training and pacing strategies, by examining the effects of the preceding exercise on subsequent running under laboratory conditions (Walsh et al., 2017; Bonacci et al., 2010; Chapman et al., 2008; Bonacci et al., 2013; Gottschall \& Palmer, 2000; Hue et al., 1999). However, despite the fact that knowledge of performance under competition is important for athletes and coaches, little has been reported on performance decrement in TR compared with IR, and inter-individual differences, under such conditions. Examining the effect of performance decrement in TR compared with IR on overall triathlon under competition will help to clarify how important the cycle to run transition training is for triathlon success.

This study aimed to clarify the decrease in TR performance compared to IR performance and the degree at which it changes. Based on physiological and biomechanical changes following preceding exercise, we hypothesise that performance in TR decreases significantly when compared with IR. We further hypothesised that these performance decreases vary widely among individuals because of differences in pacing strategy, drafting conditions, triathlon experience, and performance level (Hausswirth et al., 2010; Hauswirth et al., 2001; Rendos et al., 2013; Millet et al., 2000)..

\section{Materials and Methods}

Experimental Approach to the Problem

Data were obtained from the website (http://www.jtu.or.jp/) for official result times of certification competition events (IR) from 2013 to 2016 in the Japan Triathlon Union, and elite categories participating in the Asia Cup Osaka sprint triathlon (TR) from 2013 to 2016 in the International Triathlon Union.

\section{Subjects and Race information}

The data of 236 athletes who finished the IR and TR in the same year were analysed (2013; male $\mathrm{n}=37$, female $\mathrm{n}=19$, 2014; male $\mathrm{n}$ $=52$, female $\mathrm{n}=19$, 2015; male $\mathrm{n}=28$, female $\mathrm{n}=26$, 2016; male $\mathrm{n}=36$, female $\mathrm{n}=19$ ). Because these data are in the public domain and is freely available on the internet, no formal ethics committee approval was necessary.

IR was a $5-\mathrm{km}$ run on a $400-\mathrm{m}$ outdoor track. TR was a $5-\mathrm{km}$ running preceded by $0.75-\mathrm{km}$ swimming and $20-\mathrm{km}$ cycling on a flat road surface. Each race condition is shown in Table 1. The interval between IR and TR was within six months. The transition times in each year, were not explicitly listed.

\section{Procedures}

The IR data analysed were the athlete's season-best times. To evaluate the degree of change in the performance of TR compared 
to IR, the rate of change in performance (\%) was calculated as follows:

rate of change in performance $(\%)=((\rrbracket$

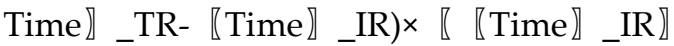
$\wedge(-1)) \times 100$

where 『Time】_TR is the running time in the triathlon and 『Time】_IR is the IR time. The performance of TR in comparison with IR is inferior when the value is positive and is better when the value is negative.

\section{Statistical Analyses}

All values are presented as the mean \pm standard deviation (SD). Data analyses were conducted using SPSS 22.0 (SPSS, Inc., Chicago, IL, USA). Although the analysed races had the same course profile, the environment and race development varied each year. The data were therefore analysed for every year. To compare differences in performance between IR and TR, a paired Wilcoxon test was used. The effect sizes $(r)$ were calculated as the $z$ /square $\operatorname{root}(\mathrm{N})(\mathrm{N}$, number of observations) (Fritz \& Morris, 2012) and interpreted as very small $(<0.1)$, small $(\geq 0.1$ and $<0.3)$, moderate $(\geq 0.3$ and $<0.5)$, or large $(\geq 0.5)$ (Cohen, 1988).

To assess whether the change in running performance influences triathlon success, Spearman's rank correlation was used to determine the relationship between TR and IR performance, TR performance and rate of change in performance, and the overall triathlon performance and rate of change in performance. Spearman's rank correlation coefficients (rho) were interpreted as very small $(<0.1)$, small $(\geq 0.1$ and $<0.3)$, moderate $(\geq 0.3$ and $<0.5)$, or large $(\geq 0.5)$ (Cohen, 1988). Statistical significance was set at $p<0.05$.

\section{Results}

Tables 2 and 3 show the average IR and TR performance and the rate of change in performance for each competition event. The average performance was significantly worse in the TR than in the IR, and the effect size in the paired Wilcoxon test was large, regardless of competition years and sex $(\mathrm{p}<$ $0.01)$. Tables 2 and 3 also show that large inter-individual responses were evident. For example, the rate of change in performance in men in 2014 was in the range of from 2.5 to $69.5 \%$.

The TR performance was significantly correlated with IR performance $(\mathrm{p}<0.01$, rho $=0.48-0.78$; Table 3$)$ and the rate of change in performance $(\mathrm{p}<0.01$, rho $=0.67-0.93$; Table 4 ), regardless of the year of the competition or sex. The overall triathlon performance significantly correlated with the rate of change in performance, except for women athletes in $2016(\mathrm{p}<0.05$, rho $=0.47-0.76$; Table 4).

\section{Discussion}

This study investigated the decrease, and degree of change in TR performance compared with IR performance. The performance of TR compared to IR proved inferior, and the degree of change varied widely inter-individual. To our knowledge, this study is the first to report the performance decrement in TR compared with IR for competitive events with large datasets.

When examining the TR performance in comparison with the IR performance (Table 2), it is generally assumed that the decrease in performance following the cycling exercise was due to deterioration of physiological status (increases in VO2, VE, $\mathrm{HR}$, breathing frequency, BLa concentration, $\mathrm{VO} 2$ of the respiratory muscles, and core temperature) and biomechanical changes (increase in muscle activity, leg stiffness and changes in running kinematics). This assumption is supported by numerous studies (Hue et al., 2000; Taylor \& Smith, 2013; Walsh et al., 2017; Walsh et al., 2015; Millet et al., 2000; Walsh et al., 2017; Le Meur et al., 2012; Rendos et al., 2013). However, these studies specifically investigated physiological and biomechanical changes following the cycling exercise under laboratory conditions, with controlled environmental factors (Hue et al., 2000; Walsh et al., 2017; Walsh et al., 2015; Millet et al., 2000; Walsh et al., 2017; Le 
Meur et al., 2012; Rendos et al., 2013). Taylor and Smith (2013) indicated that the performance of TR decreased by $1 \mathrm{~min} 21 \mathrm{~s}$ (6.9\%) compared with IR under controlled environmental factors, whereas in our study, the decrease in TR performance under competition conditions ("from $1 \mathrm{~min}$ $15 \mathrm{~s}$ to $4 \min 24 \mathrm{~s}$ ), proved to be greater than what was reported in the controlled environment study. It can be concluded that various extrinsic factors (i.e. temperature, humidity, course profile, race development, and competitor profile) may influence the decrement in TR performance. For example, the decrement performance in TR compared with IR in male triathletes in 2015 was much greater than that in the other races evaluated (Table 2). Note that race condition temperatures in $2015\left(31.3^{\circ} \mathrm{C}\right)$ were the highest recorded of all the races evaluated (Table 1). Chan et al. (2008) reported that triathletes reduced their running performance after cycling, when the ambient temperature was high. It therefore appears that high temperatures influence the decrease in TR performance.

The sex of triathletes must also be considered. In the data evaluated, there was a clear tendency for women to show decreased performance to a lesser extent than men (Tables 2 and 3). The reasons for this are as follows: First, muscle fatigue is different between men and women. Women have been shown to have greater relative fatigue resistance than males (Hicks et al., 2001). Second, Le Meur et al. (2009) indicated that men and women adopt different pacing strategies during the cycling phase. Male athletes were inclined to push the pace during the cycling phase and to therefore reach high power output bursts. Consequently, residual fatigue induced by preceding exercise can differ between men and women.

There was a significant correlation between overall triathlon performance and rate of change in performance (Table 4). This relationship indicates that triathletes with a higher performance level tend to show lower performance decreases. Millet et al.
(2000) reported that sub-elite triathletes show more adverse running economy alterations during running after cycling, than elite triathletes. Bonacci et al. (2011) also demonstrated that cycling did not change the running economy in an elite triathlete group that included Olympians and the World Champion. Running economy is one of the major determinants of distance running performance (Saunders et al., 2004). These previous studies (Millet et al., 2000; Bonacci et al., 2011) support our finding that performance decrement in TR relates to performance level.

Also important to note is that large inter-individual responses were evident in the results of the performance decrement in TR when compared with IR (Table 2,3). Bonacci et al. (2010) conducted a study to compare IR and running after 45 minutes of high intensity cycling and reported that seven out of 15 triathletes demonstrated changes in muscle activity and running kinematics, such as the angle of the ankle at foot contact. Moreover, eight out of 15 triathletes demonstrated an increase or decrease in running economy during running after cycling, compared with IR. These authors argue that the effects of cycling on neuromuscular control during subsequent running are individualised. Chapman et al. (2008) reported that muscle activity was different between IR and running after 20 minutes of moderately constant power output cycling, in five out of 14 triathletes, and in 10 out of 34 triathletes (Chapman et al., 2010). Several other studies (Bonacci et al., 2010; du Plessis et al., 2020; Rendos et al., 2013) indicate that the change in running economy and running form as determinants of successful distance running performance, is a factor of inter-individual differences, which may explain the interindividual differences in the degree of performance decrement in TR.

In real competition, pacing strategy would also differ between triathletes. One of the characteristic factors influencing pacing strategy in a triathlon is drafting during cycling (Wu et al., 2014). The presence or 
absence and frequency of drafting during cycling differed between triathletes in the present study, due to draft-legal races. A study of the effects of drafting during cycling on subsequent running, indicated that $\mathrm{VO} 2, \mathrm{VE}$, and $\mathrm{HR}$ during cycling were higher for no-draft cycling than for drafting cycling (Hausswirth et al., 1999). In addition, performance in the subsequent running was inferior for no-draft cycling compared with drafting cycling (Hausswirth et al., 1999; Hausswirth et al., 2001). Taken together, the pacing strategy most likely influences interindividual differences in the performance decrement in TR. Future studies should be conducted to understand how pacing strategy influences performance decrement in TR.

TR performance is more important than swimming or cycling performance to overall triathlon success (Le Meur et al., 2009; Vleck et al., 2008; Vleck et al., 2006). Strategies for improving TR performance include improving IR performance and minimally decreasing TR versus IR performance. In the present study, TR performance was significantly correlated with IR performance $(\mathrm{p}<0.01$, rho $=0.48-0.78$; Table 4$)$ and the rate of change in performance $(\mathrm{p}<0.01$, rho $=0.67-0.93$; Table 4). This result implies that minimal performance decrement in TR compared with IR is essential for improving TR performance, as with improving IR performance. The average decrease in TR compared to IR performance ranged between $1 \mathrm{~min} 15 \mathrm{~s}$ and $4 \mathrm{~min} 24 \mathrm{~s}$ in triathletes (Table 2,3). Considering that the differential time at the end between the top 10 triathletes during Sprint World Triathlons is shorter than $1 \mathrm{~min}$

(https://www.triathlon.org/results/), minimal performance decrement in TR compared with IR is important for overall triathlon success. Moreover, although the overall triathlon results are affected by multiple variables, the overall triathlon performance and the rate of change in performance showed significant correlation $(\mathrm{p}<0.05$, rho $=0.47-0.76$; Table 4$)$.
Our study has several limitations. First, with triathlon events, extrinsic factors (i.e. temperature, humidity, course profile, race development, and competitor profile) influence performance. In the present study, the running courses for either IR or TR competitions were also flat. In other race conditions, the decrease in TR performance and inter-individual differences may be similar to or substantially different to those indicated by our results. The performance decrement in TR compared with IR is likely to be overestimated because the recorded temperatures in TR competitions were higher than in IR competitions (Table 1). Second, our results do not consider the transition time between cycling and running events. The mean transition time is $28 \mathrm{~s}$ for standard distance triathlons (Cejuela et al., 2013). The transition time in sprint distance triathlons will be similar to or shorter than that in standard distance. However, the presence or absence of the transition times or variability of these times will most likely not lead to different conclusions regarding the decrease in TR performance. Third, the present study could not identify the effect of pacing strategy during preceding exercise on the performance decrement in TR compared with IR. The pacing strategy during preceding exercise does influence the TR performance (Le Meur et al., 2009, Hausswirth et al., 2010). Therefore, further research is needed to confirm the impact of pacing strategy during preceding exercise on inter-individual responses.

In conclusion, TR performance decreases compared with IR performance, and the degree of change, varies widely interindividual in sprint triathlons. Moreover, the degree of decrease in TR performance does affect the total triathlon performance. Our findings showed that the smaller the performance decrement in TR compared with IR, the more likely the athlete will be to succeed in the sprint triathlon.

\section{Practical Applications.}

Training and competitive race strategies that minimise the performance 
decrement in TR compared with IR are recommended for athletes, given that the degree of decrease in performance influences triathlon success. It is important to prioritise training that minimises performance decrement in TR compared with IR, especially for athletes who show large decreases in TR performance.

Acknowledgments: We would like to thank 'Editage' (www.editage.jp) for English language editing.

Conflicts of Interest: The authors declare that they have no conflict of interest.

\section{References}

1. Bonacci J, Green D, Saunders PU, Blanch P, Franettovich M, Chapman AR, \& Vicenzino $B$ (2010) Change in running kinematics after cycling are related to alterations in running economy in triathletes. Journal of Science and Medicine in Sport 13 (4): 460-464.

2. Bonacci J, Saunders PU, Alexander M, Blanch P, \& Vicenzino B (2011) Neuromuscular control and running economy is preserved in elite international triathletes after cycling. Sports Biomechanics 10 (1): 59-71.

3. Bonacci J, Vleck V, Saunders PU, Blanch P, \& Vicenzino B (2013) Rating of perceived exertion during cycling is associated with subsequent running economy in triathletes. Journal of Science and Medicine in Sport 16 (1): 49-53.

4. Cejuela R, Cala A, Perez-Turpin JA, Villa JG, Cortell JM, \& Chinchilla JJ (2013) Temporal activity in particular segments and transitions in the Olympic triathlon. Journal of Human Sport and Exercise 36, 87-95.

5. Chan KOW, Wong AHA, Chen YJ (2008) Effects of a hot environment on simulated cycling and running performance in triathletes. The Journal of Sports Medicine and Physical Fitness 48 (2): 149-157.

6. Chapman AR, Hodges PW, Briggs AM, Stapley PJ, \& Vicenzino B (2010) Neuromuscular control and exercise-related leg pain in triathletes. Medicine and Science in Sports and Exercise 42 (2): 233-243.
7. Chapman AR, Vicenzino B, Blanch P, Dowlan S, \& Hodges PW (2008) Does cycling effect motor coordination of the leg during running in elite triathletes? Journal of Science and Medicine in Sport 11 (4): 371-380.

8. Cohen J (1988) Statistical power analysis for the behavioral sciences. 2nd ed. L. Erlbaum Associates.

9. du Plessis C, Blazevich AJ, Abbiss C, Wilkie JC (2020) Running economy and effort after cycling: effect of methodological choices. Journal of Sports Sciences, 38 (10): 1105-1114.

10. Fritz CO, \& Morris PE (2012) Effect size estimates: current use, calculations, and interpretation. Journal of Experimental Psychology-General 141 (1): 2-18.

11. Gottschall JS, \& Palmer BM (2000) Acute effects of cycling on running step length and step frequency. The Journal of Strength and Conditioning Research 14 (1): 97-101.

12. Hausswirth C, Lehenaff D, Dreano P, \& Savonen K (1999) Effects of cycling alone or in a sheltered position on subsequent running performance during a triathlon. Medicine and Science in Sports and Exercise 31 (4): 599-604.

13. Hausswirth C, Le Meur Y, Bieuzen F, Brisswalter J, Bernard T (2010) Pacing strategy during the initial phase of the run in triathlon: influence on overall performance. European Journal of Applied Physiology 108 (6): 1115-1123.

14. Hausswirth C, Vallier JM, Lehenaff D, Brisswalter J, Smith D, Millet G, \& Dreano (2001) Effect of two drafting modalities in cycling on running performance. Medicine and Science in Sports and Exercise 33 (3): 485-492.

15. Hicks AL, Kent-Braun J, Ditor DS (2001) Sex differences in human skeletal muscle fatigue. Exercise and Sport Sciences Reviews 29 (3): 109-112.

16. Hue O, Gallais DL, Boussana A, Chollet D, \& Prefaut C (2000) Performance level and cardiopulmonary responses during a cyclerun trial. International Journal of Sports Medicine 21 (4): 250-255.

17. Hue O, Gallais DL, Boussana A, Chollet D, \& Prefaut C (1999) Ventilatory responses during experimental cycle-run transition in triathletes. Medicine and Science in Sports and Exercise 31 (10): 1422-1428. 
18. Le Meur Y, Dorel S, Rabita G, Bernard T, Brisswalter J, \& Hausswirth C (2012) Springmass behavior and electromyographic activity evolution during a cycle-run test to exhaustion in triathletes. Journal of Electromyography Kinesiology 22 (6): 835844.

19. Le Meur Y, Hausswirth C, Dorel S, Bignet F, Brisswalter J, \& Bernard T (2009) Influence of gender on pacing adopted by elite triathletes during a competition. European Journal of Applied Physiology 106 (4): 535-545.

20. Millet GP, Millet GY, Hofmann MD, \& Candau RB (2000) Alterations in running economy and mechanics after maximal cycling in triathletes: Influence of performance level. International Journal of Sports Medicine 21 (2): 127-132.

21. Millet GP, \& Vleck VE (2000) Physiological and biomechanical adaptations to the cycle to run transition in Olympic triathlon: review and practical recommendations for training. British Journal of Sports Medicine 34 (5): 384-390.

22. Rendos NK, Harrison BC, Dicharry JM, Sauer LD, \& Hart JM (2013) Sagittal plane kinematics during the transition run in triathletes. Journal of Science and Medicine in Sport 16 (3): 259-265.

23. Saunders PU, Pyne DB, Telford RD, \& Hawley JA (2004) Factors affecting running economy in trained distance runners. Sports Medicine 34 (7): 465-485.

24. Sleivert GG, \& Rowlands DS (1996) Physical and physiological factors associated with success in the triathlon. Sports Medicine 22 (1): 8-18.

25. Taylor D, \& Smith MF (2013) Scalar-liner increases in perceived exertion are dissociated from residual physiological responses during sprint-distance triathlon. Physiology and Behavior 118 (13): 178-184.

26. Vleck VE, Bentley DJ, Millet GP, \& Bürgi A (2008) Pacing during an elite olympic distance triathlon: comparison between male and female competitors. Journal of Science and Medicine in Sport 11 (4): 424-432.

27. Vleck VE, Bürgi A, \& Bentley DJ (2006) The consequences of swim, cycle, and run performance on overall result in elite olympic distance triathlon. International Journal of Sports Medicine 27 (1): 43-48.
28. Walsh JA, Dawber JP, Lepers R, Brown M, \& Stapley PJ (2017) Is moderate intensity cycling sufficient to induce cardiorespiratory and biomechanical modifications of subsequent running? The Journal of Strength and Conditioning Research 31 (4): 1078-1086.

29. Walsh JA, Stamenkovic A, Lepers R, Peoples G, \& Stapley PJ (2015) Neuromuscular and physiological variables evolve independently when running immediately after cycling. Journal of Electromyography Kinesiology, 25 (6): 887-893.

30. Wu SS, Peiffer JJ, Brisswalter J, Nosaka K, Abbiss CR (2014) Factors influencing pacing in triathlon. Open Access Journal of Sports Medicine, 5, 223-234. 


\section{Tables}

Table 1. Race conditions

\begin{tabular}{rccc}
\hline & Temperature $\left({ }^{\circ} \mathbf{C}\right)$ & Relative humidity (\%) & Wind speed (m/s) \\
\hline TR 2013 & 29.4 & 56.3 & 0.8 \\
2014 & 28.4 & 72.3 & 1.5 \\
2015 & 31.3 & 58.2 & 0.0 \\
2016 & 30.6 & 69.0 & 1.5 \\
Mean \pm SD & $29.9 \pm 1.3$ & $64.0 \pm 7.9$ & $0.9 \pm 0.7$ \\
IR 2013 & $12.8 \pm 7.6$ & $60.0 \pm 16.1$ & $4.7 \pm 2.6$ \\
2014 & $16.7 \pm 5.5$ & $41.8 \pm 9.3$ & $4.5 \pm 2.4$ \\
2015 & $17.8 \pm 7.8$ & $42.9 \pm 15.4$ & $3.7 \pm 1.7$ \\
2016 & $15.5 \pm 5.7$ & $50.9 \pm 20.8$ & $3.2 \pm 2.1$ \\
Mean \pm SD & $15.7 \pm 2.2$ & $48.9 \pm 8.4$ & $4.0 \pm 0.7$
\end{tabular}

Note: $\mathrm{TR}=$ running preceded by swimming and cycling in the Asia Cup Osaka sprint triathlon competition, IR = running without prior swimming and cycling in the certification competitive events which were held in all over Japan (Tokyo, Fukushima, Fukui, Tochigi, Saitama, Chiba, Kanagawa, Yamanashi, Shizuoka, Aichi, Kyoto, Osaka, Hyogo, Tottori, Hiroshima, Ehime, Fukuoka, Miyazaki).

Table 2. Change in TR compared to IR performance and variability in male triathletes

\begin{tabular}{ccccc}
\hline & & Mean \pm SD & Min - Max & Effect size \\
\hline Male 2013 & TimeIR & $0: 16: 20 \pm 0: 00: 41$ & $0: 15: 13-0: 18: 00$ & \\
$(\mathrm{n}=37)$ & TimeTr & $0: 18: 34 \pm 0: 01: 26^{* *}$ & $0: 16: 22-0: 22: 30$ & 0.87 (large) \\
& Rate of change (\%) & $13.6 \pm 7.4$ & $3.5-34.3$ & \\
Male 2014 & TimeIR & $0: 16: 46 \pm 0: 01: 00$ & $0: 15: 19-0: 18: 54$ & \\
$(\mathrm{n}=52)$ & TimeTR & $0: 20: 02 \pm 0: 01: 38^{* *}$ & $0: 17: 50-0: 26: 11$ & 0.87 (large) \\
& Rate of change (\%) & $19.7 \pm 10.1$ & $2.5-69.5$ & \\
Male 2015 & TimeIR & $0: 15: 55 \pm 0: 00: 35$ & $0: 15: 06-0: 17: 27$ & \\
$(\mathrm{n}=28)$ & TimeTR & $0: 20: 19 \pm 0: 02: 06^{* *}$ & $0: 17: 19-0: 27: 17$ & 0.87 (large) \\
& Rate of change (\%) & $27.7 \pm 12.5$ & $13.4-72.1$ & \\
Male 2016 & TimeIR & $0: 16: 02 \pm 0: 00: 35$ & $0: 15: 15-0: 17: 15$ & \\
$(\mathrm{n}=36)$ & TimeTr & $0: 18: 10 \pm 0: 01: 31^{* *}$ & $0: 15: 41-0: 20: 45$ & 0.87 (large) \\
& Rate of change (\%) & $13.2 \pm 7.7$ & $2.5-28.3$ &
\end{tabular}

Note: Effect size = the effect size in a paired Wilcoxon test (i.e. r), Rate of change $=$ the change in TR compared to IR performance $(\%)$, TimeIR = time in isolated running (h:min:s), TітетR = time in triathlon running (h:min:s); Significantly different from TimeIR, ${ }^{* *} \mathrm{p}<0.01$ 
Table 3. Change in TR compared to IR performance and variability in female triathletes

\begin{tabular}{|c|c|c|c|c|}
\hline & & Mean \pm SD & Min - Max & Effect size \\
\hline Female 2013 & TimeIr & 0:19:06 \pm 0:01:05 & $0: 17: 38-0: 20: 52$ & \\
\hline \multirow[t]{2}{*}{$(\mathrm{n}=19)$} & Тіметr & $0: 20: 55 \pm 0: 01: 34^{* *}$ & $0: 18: 21-0: 23: 44$ & 0.88 (large) \\
\hline & $\begin{array}{c}\text { Rate of change } \\
(\%)\end{array}$ & $9.5 \pm 5.1$ & $1.6-22.8$ & \\
\hline Female 2014 & TimeIr & $0: 18: 43 \pm 0: 01: 32$ & $0: 17: 05-0: 22: 52$ & \\
\hline \multirow[t]{2}{*}{$(\mathrm{n}=19)$} & Тіметr & $0: 21: 55 \pm 0: 02: 16^{* *}$ & 0:19:09 - 0:28:15 & 0.88 (large) \\
\hline & $\begin{array}{c}\text { Rate of change } \\
(\%)\end{array}$ & $17.0 \pm 6.0$ & $7.0-27.4$ & \\
\hline Female 2015 & TimeIr & 0:19:00 \pm 0:01:07 & $0: 17: 06$ - 0:21:57 & \\
\hline \multirow[t]{2}{*}{$(\mathrm{n}=26)$} & Тіметr & $0: 22: 14 \pm 0: 01: 48^{* *}$ & $0: 19: 13-0: 25: 13$ & 0.87 (large) \\
\hline & $\begin{array}{c}\text { Rate of change } \\
(\%)\end{array}$ & $17.1 \pm 6.7$ & $8.1-35.8$ & \\
\hline Female 2016 & TimeIr & $0: 18: 43 \pm 0: 00: 59$ & $0: 17: 10$ - 0:20:21 & \\
\hline \multirow[t]{2}{*}{$(\mathrm{n}=19)$} & Тіметr & $0: 19: 58 \pm 0: 01: 40^{* *}$ & $0: 17: 42$ - 0:23:34 & 0.88 (large) \\
\hline & $\begin{array}{c}\text { Rate of change } \\
(\%)\end{array}$ & $6.7 \pm 6.3$ & $0.5-23.3$ & \\
\hline
\end{tabular}

Table 4. Spearman correlation analysis of TR performance, IR performance, rate of change in TR compared to IR performance, and overall triathlon performance

\begin{tabular}{|c|c|c|c|}
\hline Event & Тіметr vs Timerr & $\begin{array}{c}\text { Timetr vs Rate of } \\
\text { change }\end{array}$ & $\begin{array}{l}\text { Timeoot vs Rate of } \\
\text { change }\end{array}$ \\
\hline Male $2013(n=37)$ & 0.63 (large) $^{* *}$ & 0.79 (large) $^{* *}$ & 0.47 (moderate) $^{* *}$ \\
\hline Male $2014(n=52)$ & $0.52(\text { large })^{* *}$ & $0.68(\text { large })^{* *}$ & $0.51(\text { large })^{* *}$ \\
\hline Male $2015(n=28)$ & 0.48 (moderate) $^{* *}$ & $0.89{\text { (large })^{* *}}^{*}$ & $0.70(\text { large })^{* *}$ \\
\hline Male $2016(n=36)$ & $0.64{\text { (large })^{* *}}^{*}$ & $0.93{\text { (large })^{* *}}^{*}$ & $0.76(\text { large })^{* *}$ \\
\hline Female $2013(n=19)$ & 0.77 (large $)^{* *}$ & $0.73(\text { large })^{* *}$ & $0.65{\text { (large })^{* *}}^{*}$ \\
\hline Female $2014(n=19)$ & $0.78{\text { (large })^{* *}}^{* *}$ & 0.67 (large) $^{* *}$ & 0.53 (large) $^{*}$ \\
\hline Female $2015(n=26)$ & 0.71 (large) $)^{* *}$ & $0.82(\text { large })^{* *}$ & $0.63(\text { large })^{* *}$ \\
\hline Female $2016(n=19)$ & $0.77{\text { (large })^{* *}}^{* *}$ & $0.67{\text { (large })^{* *}}^{* *}$ & 0.24 (small) \\
\hline
\end{tabular}

Note: Rate of change $=$ the change in TR compared to IR performance, TimeIR $=$ time in isolated running, Timeot $=$ time in overall triathlon time, TimeтR $=$ time in triathlon running; Significantly relationship, ${ }^{*} \mathrm{p}<0.05,{ }^{* *} \mathrm{p}<0.01$ 